\title{
HIGH RESOLUTION AEROSOL OPTICAL DEPTH MAPPING OF BEIJING USING LANSAT8 IMAGERY
}

\author{
Yan $\mathrm{Li}^{*}$, Yuanliang Liu, Jianliang Wu \\ International Institute for Earth System Science, Nanjing University, Jiangsu Provincial Key Laboratory of Geographic Information \\ Science and Technology, China - liyan@nju.edu.cn, (915214453, 1595941721)@qq.com
}

THS-18

KEY WORDS: AOD, Retrieval, Landsat8, Dark target, Impervious surface

\begin{abstract}
:
Aerosol Optical Depth (AOD) is one of the most important parameters in the atmospheric correction of remote sensing images. We present a new method of per pixel AOD retrieval using the imagery of Landsat8. It is based on Second Simulation of the Satellite Signal in the Solar Spectrum (6S). General dark target method takes dense vegetation pixels as dark targets and derives their 550nm AODs directly from the LUT, and interpolates the AODs of other pixels according to spatial neighbourhood using those of dark target pixels. This method will down estimate the AOD levels for urban areas. We propose an innovative method to retrieval the AODs using multiple temporal data. For a pixel which has nothing change between the associated time, there must exists an intersection of surface albedo. When there are enough data to find the intersection it ought to be a value that meet the error tolerance. In this paper, we present an example of using three temporal Landsat ETM+ image to retrieve AOD taking Beijing as the testing area. The result is compared to the commonly employed dark target algorithm to show the effectiveness of the methods.
\end{abstract}

\section{INTRODUCTION}

\subsection{Research Background of AOD Retrieval}

An aerosol is a colloid of fine solid particles or liquid droplets in air or another gas(Hinds, 1999). Aerosol particles with radius less than $10 \mu \mathrm{m}$ (PM10) can get into one's bronchial respiratory system, and threaten one's health. Due to the strong scattering and absorbing effects to the visible light, aerosol particles with radius less than $2.5 \mu \mathrm{m}$ (PM2.5) will reduce the visibility, cause the haze and introduce troubles to the traffic. AOD is defined as the integrated extinction coefficient over a vertical column of unit cross section. AOD is one of the most important parameters in the atmospheric correction of remote sensing images, which is the key factor for correction accuracy. In the traditional atmospheric correction for remote sensing imagery, AOD is generally taken as an uniformed parameter regardless of its spatial distribution variance. This assumption will cause the decreasing accuracy for the correction and result in bad quantification for the remote sensing studies. It is clear that correct retrieval of the AOD is the prerequisite for high accuracy and high correction retrieval of the land surface parameters.

\subsection{Related Researches}

The retrieval of AOD using remote sensed data has been developed for many years. Considering the diversity in aerosol types, we are only interested in the continental aerosol retrieval. Besides of the newly developed numerical calculation methods, such as data mining (Vucetic, 2008), the methods based on radiative transfer models can be catalogued into the following classes.

Dark Target (DT) Algorithm (Kaufman, 1997a; Remer, 2005; Chu,2002; Ichoku, 2002; Levy, 2005) is a widely applied method which is mainly adopted for the AOD retrieval for continental aerosol. It is based on the observation that, in the areas of low surface albedo, the albedo is approximately proportional to AOD. By choosing proper aerosol model, the AOD can be retrieved using the surface albedo of red or blue light and a lookup table (LUT) derived from radiative transfer models (MODTRAN, 6S) (Vermote, 1997). Although this is the most common method for AOD retrieval of continental aerosol, it is available for lower albedo pixels, see, $\rho 2.13 \leq 0.15$ of dark pixels. It also requires the aerosol type and other priors (Kaufman, 1997b).

"Deep Blue" (DB) algorithm (Hsu, 2004; Hsu, 2006; Misra, 2015) is another popular method for AOD retrieval. It utilizes the phenomenon of the strong air reflectance and the weak surface reflectance in the blue spectrum. Supposing that the surface albedo remains unchanged in a certain period of time, the SA of good day is used to retrieve the AOD. A surface reflectance database of surface reflectivity is generated at some certain channels using minimum reflectivity technique. Finally, by matching the lookup table, the aerosol optical depth and SA are derived using two or three channels' apparent reflectance by the maximum likelihood method. DB algorithm have successful applications in Sahara Desert and Arabian Peninsula reveal various dust sources.

Our study found that DB algorithm has lower accuracy than DT by referencing the in situ AOD data of AERONET. However DT algorithm rely on interpolation to estimate the AOD on the impervious surface. No prior knowledge about the surface is applied in the interpolation. It is equalized with taking all the pixels as same as the dark targets, the vegetations. This will cause error in estimating the AOD levels for urban areas. To accurate retrieval and less blind selection for the dark targets, we propose a different method to retrieval the AODs using multiple temporal data. 


\section{METHODS}

\subsection{Simulation of AOD, Apparent reflectance, and Surface albedo}

Same with the common algorithm, assume that the underlying surface is Lambertian and homogeneous. Apparent reflectance at the top of the atmosphere $\rho_{T O A}$ received by satellite can be represented by

$$
\rho_{\mathrm{TOA}}\left(\mu, \varphi, \mu_{0}, \varphi_{0}\right)=\rho_{a}\left(\mu, \varphi, \mu_{0}, \varphi_{0}\right)+\frac{T(\mu) T\left(\mu_{0}\right) \rho_{\text {surf }}}{1-\rho_{\text {surf }} S}
$$

Equation (1) is the radiative transfer function under the ideal situation. The first term in the right is the reflectance contributed by the path, while the second term is the reflectance contributed by surface. $T(\mu)$ and $T\left(\mu_{0}\right)$ can be viewed as a constant together at certain geometric parameters, it is the transmission function describing the atmospheric effect on upward and downward radiance. $\rho_{\text {surf }}$ is the surface albedo. All the augments and parameters are functions of the spectrum. From this formulation, a set of lookup tables can be generated by using the geometric parameters associated with the image.

$6 \mathrm{~S}$ radiative transfer algorithm is the important tool for atmospheric correction. It generates a lookup table by setting the proper climate type, AOD type, the geometric parameters, etc. The LUT is a simulation function for the apparent reflectance and surface albedo under various AOD values.

Figure 1 shows a typical curve family that representing the relationship between apparent reflectance and surface albedo under certain AODs. For color mapping, only several AOD values are chosen to show.

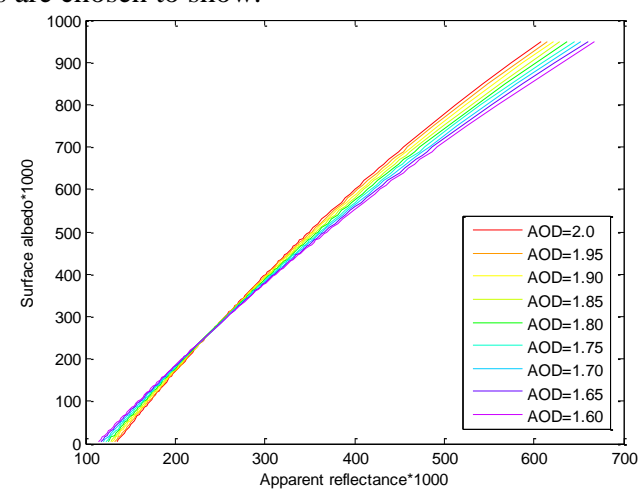

(a) 20140819

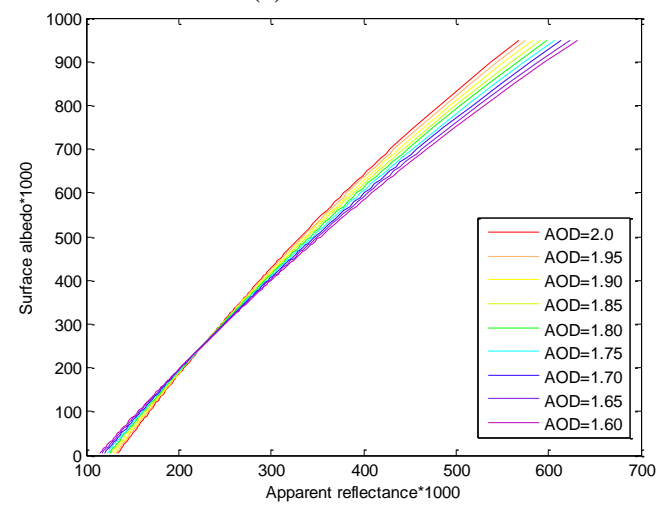

(b) 20140904
Figure 1 Apparent reflectance and surface albedo

For the same object the surface albedo is the same for various time phases, although the apparent reflectance and AOD are different. Yet, the AOD range is limited and we found that for an apparent reflectance the surface albedo is limited with a narrow band, refer to Figure 1. For a certain apparent reflectance, for example, the line at 0.4 , the range of possible surface albedo here is from 0.425 to near 0.6 .

Thus for multiple temporal data, for a pixel which has nothing change between the associated time, there must exists an intersection of surface albedo. When there are enough data to find the intersection it ought to be a value that meet the error tolerance.

\subsection{Multiple Temporal Method}

Multiple temporal method is based on the observation above. Of course, the time period where the properties of most land objects persist should be short. For time $t, t=1,2, \ldots M$. the apparent reflectance images derived from the images of the same channel of Landsat8 ETM+ are noted as $A R_{t}$. Each pixel is matched its apparent reflectance to find a nearest neighbor in the apparent reflectance column of $L U T_{t}$, and to yield a range of surface with upper bound of albedo $s a_{-} l_{t}$ and lower bound of $s a_{-} h_{t}$. For all the time $\mathrm{i}$, the intersection $\left[s a_{-} l, s a_{-} h\right]$ if there is can be found. If the range is less than 0.02 , the mean of $s a_{-} l$ and $s a \_h$ is taken as the actual surface albedo $s$ of the pixel, for all the times. For those not satisfying the creterion, a poll algorithm is developed to decide the actual surface albedo for the pixel. Poll algorithm considers the surface albedo as a Markov random field (MRF). The surface albedo of a pixel is only relative with its neighbors. Here we take a minimal neighborhood system a 3-by-3 window centered at the pixel. The maximal probability of surface albedo of the pixel is determined by the maximal voting $s$ within the ranges of the neighboring pixels. If this value is not unique, the mean of the upper bound and the lower bound is taken as the actual surface albedo if their difference is less than 0.02 .

Here the range cutoff is 0.02 because According to our former researches, the error level of albedo by DT algorithm under 0.02 occupies the $90 \%$ of pixels. Many researches also think that 0.02 error is acceptable(Yale, 2016). Obviously, it is easier to yield accurate result by using as more as possible time phases images. If available cloud free images are rare at least two or three images should be considered.

\subsection{Results and Discussion}

2.3.1 Site and Data: Beijing is the capital of the People's Republic of China. The north and west of Beijing are surrounded by mountains, and the mid and the east-west are plain. The altitudes of the mountains range from 1000 meter to 1500 meter, while the average heights range from 40 meter to 60 meter. Beijing has a monsoon-influenced humid continental climate (Pidwirny, 2011). The six districts of the central Beijing are taken as the study area.

Landsat8 is launched on February 11 2013, and its ETM+ imagery is taken as the remote sensed data source. The Operational Land Imager (OLI) sensor of Landsat8 includes refined heritage bands, along with three new bands: a deep blue band for coastal/aerosol studies, a shortwave infrared band for cirrus detection, and a Quality Assessment band. The image 
data are download from http://www.gscloud.cn/. The picture taking time and the path and row for our data is shown in Table 1.

Table 1 The information of the testing data

\begin{tabular}{rcl}
\hline Path & Row & Date \\
\hline 123 & 32 & 1-September-2013 \\
123 & 32 & 19-August-2014 \\
123 & 33 & 4-September-2014 \\
\hline
\end{tabular}

2.3.2 AOD retrieval results: The three-time data derived valid surface albedo pixels take $80.29 \%$ of all the pixels. Initial retrieved surface albedo pixels under error of 0.02 take $37.88 \%$, as shown in Figure 2(a). By accounting for the successfully interpolated pixels, the retrieved surface albedo pixels take $69.39 \%$, as shown in Figure 2(b).

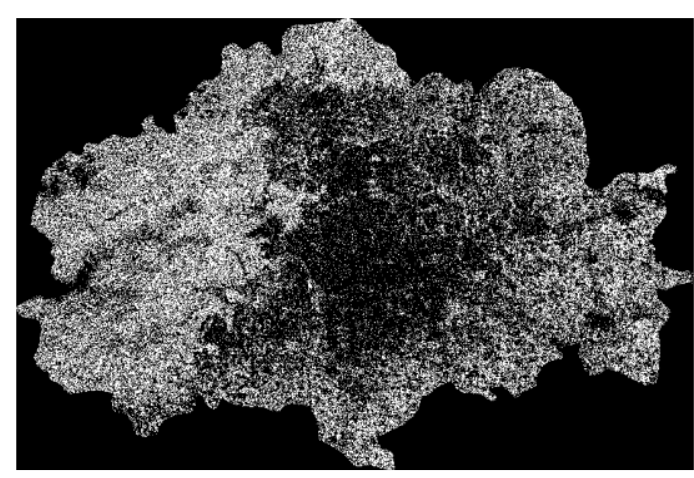

(a) error $<=0.02$

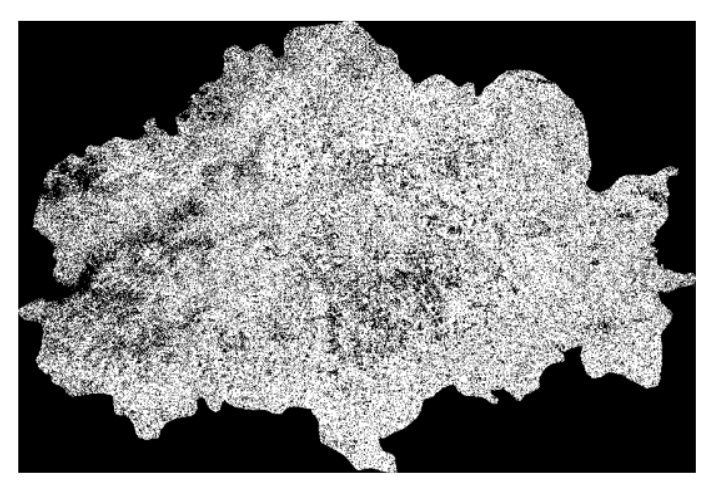

(b) all by toll

Figure 2 Intersection detection

\subsection{Comparison with Dark Target Method}

We compared the result with that derived by DT algorithm. Dark target algorithm takes dark pixels of vegetation to compute the AODs and interpolate the AOD for the remaining pixels according to spatial relevance. As example, Figure 3 (a) shows the AOD map of 19 August, 2014 by DT algorithm, and Figure 3 (b) shows the AOD map of 19 August, 2014 by our algorithm.

It can be seen that the DT algorithm down estimated the AOD in the urban areas. As we indicated in part 1.2, the interpolation by DT ignored the different surface properties. In another word, all the pixels were viewed as same as the dark targets, the vegetations, which generally cause lower AOD values.

In addition, especially for the areas of impervious surface, the AOD is more vivid and of higher sharpness than by dark target algorithm.

The exhausting evaluation of the quantitative perform of the proposed algorithm need to make in the future, where the AOD retrieved will be evaluated with respect to those inverted from the global aerosol-monitoring Aerosol Robotic Network (AERONET, http://aeronet.gsfc.nasa.gov/).

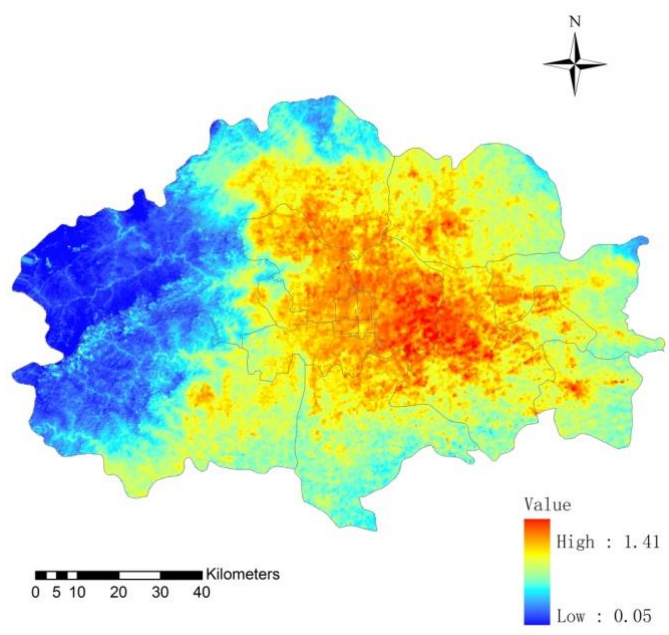

(a) Derived by DT

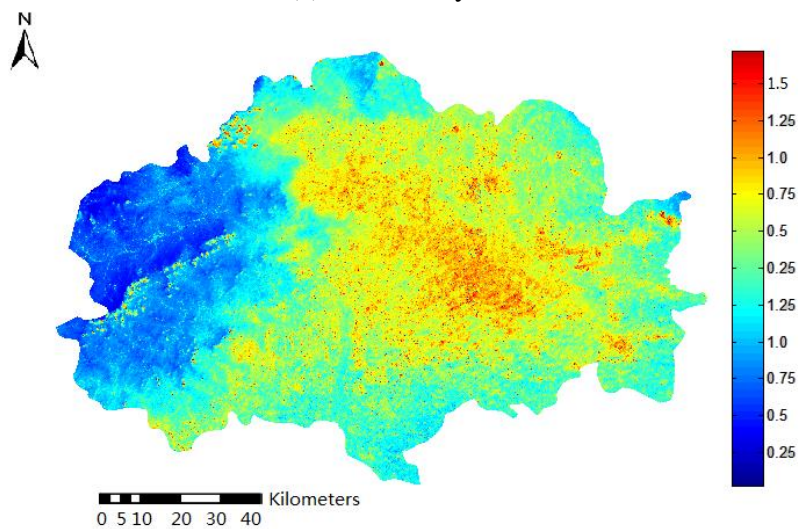

(b) Derived by our algorithm

Figure 3 AOD map of August 19, 2014

\section{CONCLUSIONS}

There is no doubt that the AOD retrieval is the most important step for atmospheric correction, and becomes widely studied field. We present a method of multiple temporal AOD retrieval technique using Landsat 8 imagery in the paper. The experiment shows its effectiveness.

The main contributions of our algorithm can be concluded as following.

1) We propose a technique of retrieving the AOD in per pixel way. It uses multiple temporal data in a short period and estimate the surface albedo and the AOD synchronously. 
2) We developed a poll algorithm to decide the maximal probability for the AOD value for the pixels that could not determine the proper value of surface albedo with satisfied error.

3) Our method gives the AOD and surface albedo for most pixels in a routine. The accuracy for the AOD compared with reference data is higher than DT. The surface albedo as the result of atmospheric correction is sharp and has better SNR than before correction.

\section{ACKNOWLEDGEMENTS}

Acknowledgements to the supports of the Chinese National Natural Science Foundation (NSFC no.41371331).

\section{REFERENCES}

Hinds, William C. (1999). Aerosol Technology (2nd ed.). Wiley - Interscience

Vucetic S., Han B., Mi W., Li Z., Obradovic Z., 2008, A DataMining Approach for the Validation of Aerosol Retrievals, IEEE Geoscience And Remote Sensing Letters, 5(1), pp. 113117.

Vermote E F , Tanré D, Deuzé J L, et al.,, 1997, Second simulation of the satellite signal in the solar spectrum, 6S:an overview, .IEEE Trans Geo \& Remote Sensing, 35(3), pp. 675686.

Kaufman, Y.J., Tanre, D., Remer, L.A., Vermote, E.F., Chu, A. Holben, B.N. 1997a, Operational Remote Sensing of Tropospheric Aerosol over Land from EOS Moderate Resolution Imaging Spectroradiometer, J. Geophys. Res. 102, 17051, doi: 10.1029/96JD03988.

Remer, L.A., Kaufman, Y.J., Tanré, D., Mattoo, S., Chu, D.A., Martins, J.V., Li, R.R., Ichoku, C., Levy, R.C., Kleidman, R.G., Eck, T.F., Vermote, E. Holben, B.N.(2005, The MODIS Aerosol Algorithm, Products, and Validation. J. Atmos. Sci. 62, pp. 947-973.

Chu, D.A., Kaufman, Y.J., Ichoku, C., Remer, L.A., Tanre, D., Holben, B.N., 2002, Validation of MODIS Aerosol Optical Depth Retrieval over Land, Geophys. Res. Lett. 29: 8007.

Ichoku, C., Chu, D.A., Mattoo, S., Kaufman, Y.J., Remer, L.A., Tanre, D., Slutsker, I., Holben, B.N., 2002, A Spatio-temporal Approach for Global Validation and Analysis of MODIS Aerosol Products, Geophys. Res. Lett. 29, pp. 8006.

Levy, R.C., Remer, L.A., Martins, J.V., Kaufman, Y.J., PlanaFattori, A., Redemann, J., Wenny, B., 2005, Evaluation of the MODIS Aerosol Retrievals over Ocean and Land during CLAMS, J. Atmos. Sci. 62, pp. 974-992.

Kaufman Y J, Wald A E, Remer L A, 1997b, The MODIS 2.1 $\mu \mathrm{m}$ channel-correlation with visible reflectance for use in remote sensing of aerosol, IEEE Transactions on Geoscience and Remote Sensing, 35(5), pp.1286-1298.

Hsu, N.C., Tsay, S.C., King, M.D., Herman, J.R., 2004, Aerosol Properties over Bright-Reecting Source Regions, IEEE Trans. Geosci. Remote Sens., 42, pp. 557-569.
Hsu C N, Tsay S C, King M D and Herman J R, 2006, Deep blue retrievals of Asian aerosol properties during ACE-Asia, IEEE Transactions on Geoscience and Remote Sensing, 44(11), pp. 3180-3195.

Misra1 A., Jayaraman A., Ganguly D., 2015, Validation of Version 5.1 MODIS Aerosol Optical Depth (Deep Blue Algorithm and Dark Target Approach) over a Semi-Arid Location in Western India, Aerosol and Air Quality Research, 15, pp. 252-262.

http://www.yale.edu/ceo/Documentation/Landsat\%208\%20ima ge\%20processing.pdf

Pidwirny, M. 2011, Köppen Climate Classification System. Retrieved from http://www.eoearth.org/view/article/162263 